\title{
Detection of humans in drone images for search and rescue operations
}

\author{
Nayee Muddin Khan Dousai \\ nayee.dousai@fer.hr \\ University of Zagreb \\ Zagreb, Croatia
}

\author{
Sven Lončarić \\ sven.loncaric@fer.hr \\ University of Zagreb \\ Zagreb, Croatia
}

\begin{abstract}
Object detection has solved many problems in different applications like monitoring security, search and rescue operations, semantic segmentation, autonomous driving and so on. Despite this huge success rate in normal ground captured images, it is still a challenging task to detect humans or any other objects from the UAV(Unmanned Aerial Vehicle) captured images due to a few challenges like pose and scale variations, weather conditions, artefacts like people wearing hats, varying attitude and camouflaged environment. In this paper, we propose a novel approach for the detection of humans in aerial images, for search and rescue operations. This method explains how to train the existing high-resolution aerial database of HERIDAL. The EfficientDET deep neural network is trained using a newly generated database to solve the human detection problem. To the best of our knowledge, the proposed method has achieved the best accuracy of $93.29 \%$ mAP compared to all existing methods. The proposed method has been compared to the system used by Croatian Mountain search and rescue (SAR) teams (IPSAR) and also with the state-of-art proposed HERIDAL database paper which is based on extracting the salient features, which has slightly worse result compared to the results of this paper.
\end{abstract}

\section{CCS CONCEPTS}

- Computing methodologies $\rightarrow$ Object detection; Neural networks; Computer vision; Artificial intelligence.

\section{KEYWORDS}

deep-learning, human detection, HERIDAL dataset, search and rescue operation

\section{INTRODUCTION}

Object detection has been considered as one of the major branch of research in computer vision for various industries and research organizations. During the last few decades, object detection has been considered as the most researched areas of computer vision. Object detection involves the detection of different types of objects,

Permission to make digital or hard copies of all or part of this work for personal or classroom use is granted without fee provided that copies are not made or distributed for profit or commercial advantage and that copies bear this notice and the full citation on the first page. Copyrights for components of this work owned by others than ACM must be honored. Abstracting with credit is permitted. To copy otherwise, or republish, to post on servers or to redistribute to lists, requires prior specific permission and/or a fee. Request permissions from permissions@acm.org.

APIT 2021, January 15-17, 2021, Bangkok, Thailand

(c) 2021 Association for Computing Machinery.

ACM ISBN 978-1-4503-8810-8/21/01.\$15.00

https://doi.org/10.1145/3449365.3449377 for instance, humans, animals, vehicles or any other daily needs as per the application. Object detection states the problem of solving where and which?, where exactly the object is located in the scene or image and which object is it. Object detection has solved many crucial computer vision problems including multiple object tracking [1][2], face detection and recognition[3] [4] [5], object segmentation [6][7][8], vehicle detection[9], and search and rescue operations [10]. By the help of these applications we have solved many existing problems like safe autonomous driving, saving the lives of humans and so on.

There has been a vast number of publications in object detection from the last couple of years in various applications like biomedical, aeronautics, automobile and military [11]. Detection of objects in normal ground-to-ground images has shown significant and promising results though the detection of objects in aerial images, which is still considered as one of the major challenges. There are a few drawbacks which have to be considered in drone images like pose and scale variations, adverse weather conditions like the presence of snow, dust, fog, low visibility, altitude, illumination, presence of artefacts like people wearing hats, camouflaged environment with trees and rocks, motion blur and the high image resolution. Some of them are shown in figure 1. Considering all these drawbacks, the designed architecture should be reliable enough to detect objects in all the situations. The existing handcrafted methods [1] [20] [21] lack in accuracy and performance when it comes to detection of humans or other objects in aerial images. In this paper, we propose a novel method based on a deep learning architecture which has proven to have better accuracy by detecting humans in the mountain search and rescue operations. Using UAVs have both pros and cons, as there are huge demand and supply of the drones in the consumer industry its ease to buy and use drones for the general public. There are few threats using drones like flying of drones near the airport can cause a collision with the flight [12] and have massive damage and the other example is the use of drones for photography in crowded places can harm the general public or spying of drones in government properties or private properties is considered as illegal [13]. Above all the cons, there are many pros using drones also like saving people and animals in rescue operations or at disaster situations [10]. One of the best example we can have is, while there was a recent fire break at Paris cathedral in 2019, the fire department of Paris has used drones to locate the location of fire break or to see if there are people struck inside the cathedral [14]. And the other example is saving people who are stuck in the mountains while hiking or skiing. 

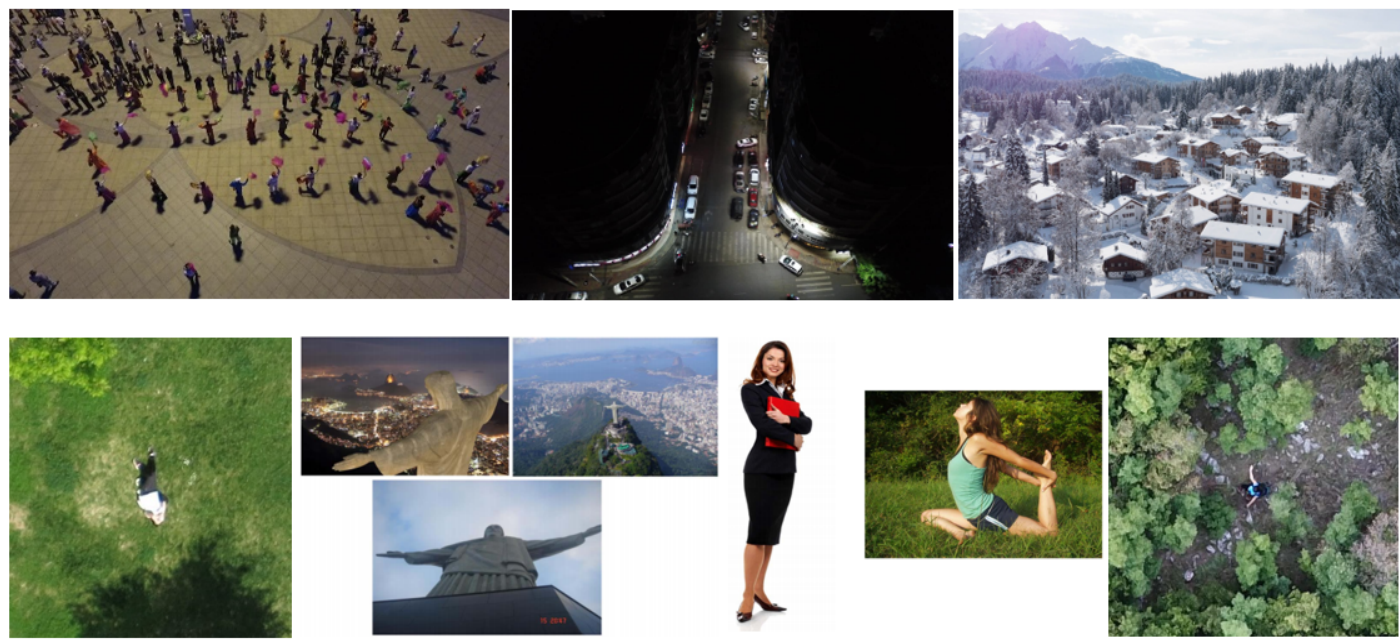

Figure 1: Few drawbacks of drone images showing scale and pose variations, low illuminance and snow and crowded images [32] [35]

\section{RELATED WORK}

There has been a vast number of papers published on object detection from the last few decades with significant accuracy but there are still very few papers on drone object detection as detection of humans from aerial images is challenging. The detection of objects in drone images has to be reliable and fast to detect the moving objects, one of the most influenced paper in real-time detection was from Viola-Jones (VJ) in 2001 [3], VJ detector has used Adaboost classifier to get the best features from the integral image by using Haar-like wavelets for speeding up the convolution process. After the evolution of VJ detectors, there were many papers which have proved to have better accuracy until the deep learning methods become more attractive in the early 2010s. Some of the papers have used the combination of handcrafted methods with hybrid deep learning methods [15] while others have implemented pure convolution neural networks (CNNs) [16] [17]. In recent papers, the authors have been using thermal imaging to detect humans based on body temperatures [18]. This type of thermal imaging methods is more suitable for the cold regions or in winter when the temperature is less than the normal human body temperature of $37^{\circ} \mathrm{C}$. When it comes to summer or tropical areas it's not the best approach as the weather conditions can be equal or more than the normal body temperature and the use of thermal imaging is also quite expensive. There has been an evolution of object detectors from the recent years after AlexNet [19] has proven to beat all the handcrafted methods like SIFT [20], HOG [21] and VJ detector [22]. Most of the deep learning architectures have been classified into two different frameworks based on region of interest (ROI)[23]. Two-stage detectors have ROI pooling layer followed by object detection and bounding box regression whereas one stage detectors have end-to-end detection without any explicitly extracting object proposals. Two-stage detectors have better accuracy like Faster RCNN [24] and FPN [25] compared to one-stage detectors while the one stage is better in time-efficient so they are mostly used in real-time object detection i.e., YOLO [26] and SSD [27].

Most of the prevalent CNNs require a fixed input size for training and testing with a small image scale which restricts from scale and aspect ratio. This is a challenging step in drone images as the drone images are usually high-resolution images of about $4000 \times 3000$. So it's really important to do some preprocessing of the images like cropping or regenerating the data set. EfficientDET [28] [29], a one-stage object detector has proven to have better efficiency and accuracy as two-stage object detectors [24] [25]. It has proposed a new scaling factor that can uniformly scale all dimensions of depth, width and resolution. By proposing a new compound coefficient and BiFPN (bi-directional feature pyramid network), EfficientNet-B7 achieves state-of-art $84.4 \%$ accuracy on ImageNet [30] and 52.2\%AP on COCO test-dev [31]. Another paper [32] has proposed a model similar to Faster RCNN [24] which uses the two-stage framework, the first stage is region proposal module which will get the salient detection of objects using morphological operations followed by CNN module to classify between the person or Non-person on HERIDAL Dataset [32]. This paper has a comparable result with Faster RCNN of $88.9 \%$.

\section{DATABASE}

Choosing Database is considered as one of the main factors in object detection as the testing results are mostly dependable on the selection of the training data. In general database is a collection of huge number of well labelled images collected from different locations and various scenarios. From the last two decades after the evolution of PASCAL VOC dataset [33] there has been alot of open source datasets available for object detection like MS-COCO [31], ImageNet [30], MNIST [34]for handwritten digits and also there are few more challenging datasets available. All these mentioned datasets have normal images collected from the daily lives or synthetic images, but for the rescue operations it's not authentic to use this type of datasets. The selection of dataset for search 


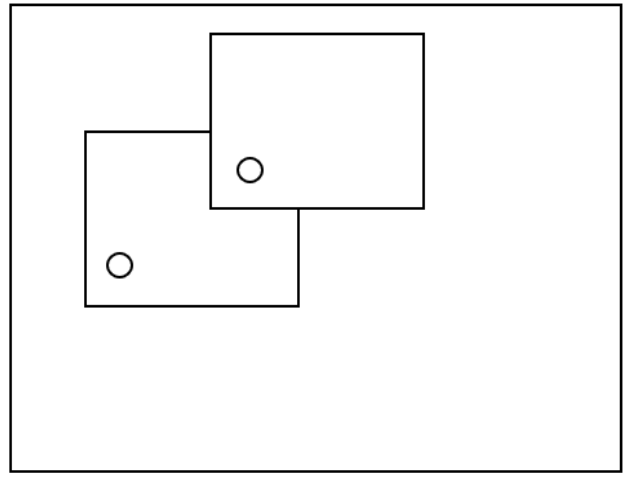

$4000 \times 3000$

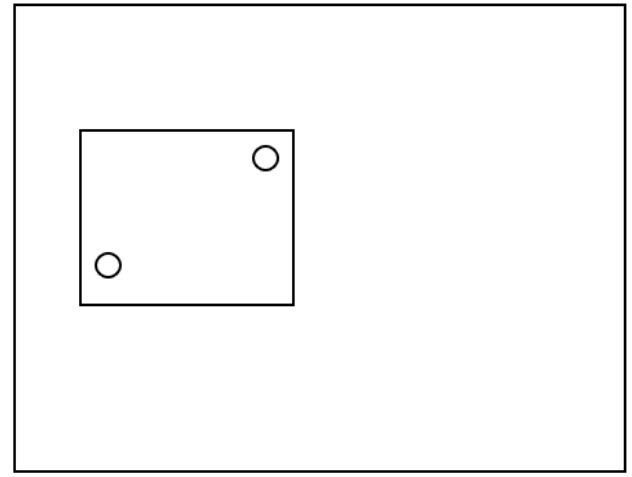

$4000 \times 3000$

Figure 2: Generating HERIDAL Dataset, Left: Two or more humans in one single image are considered as different images, Right: Two or more humans in one single image are considered as one single image

and rescue operations was the crucial part, there are very few open source datasets collected using drones like Visdrone [35] and HERIDAL [32]. Visdrone is one of the largest available database collected using drones from different cities of china. Visdrone is mainly focused on surveillance, traffic controlling or dense areas like crowded places. The drawback of this dataset is there aren't images used in search and rescue operations, so it's important for us to choose the database which has images from search and rescue operations. One of them is HERIDAL, collected from the Mediterranean and Sub-Mediterranean mountain landscapes of Bosnia. HERIDAL database contains over 68, 750 image patches with almost 1500 well-labelled training images and about 500 testing images from real world captured using drones mainly for search and rescue operation. Image patches are cropped people pictures from the real scenarios like gathered crowded places or assembled humans in a stadium. Every captured image for training and testing is $4000 \times 3000$ image resolution. All the labelled images in HERIDAL dataset is only for humans as this dataset is mainly created for search and rescue operation, by this advantage it makes us to choose this dataset for our work.

\section{PROPOSED METHOD}

One of the drawbacks with drone database is the images are usually high resolution. To overcome this drawback, it's very important to have preprocessing of the images. In our proposed method, we will generate a new dataset based on the existing HERIDAL dataset [32] HERIDAL database has $4000 \times 3000$ image pixel size which is quite complicated when we have to train them on Graphical Processing Unit (GPU) as the GPU doesn't have the high processing time with huge memory. We will generate the new database with $512 \times 512$ image resolution based on the real ground truth. The new generated database can be differentiated into two different scenarios as shown in the fig. 2. For the first scenario, If there are two or more humans within respective generating new image resolution we will include them and consider as one single image with two or more humans detected in the image. In the second scenario, when there are two or more humans within respective generating new image resolution we will consider them as different images and generate them as two or more different images. And the other factor we will keep in mind while generating the database is we will ignore the humans with less than $10 \times 10$ pixel value from the original image. Considering this two scenarios we can generate the database with various image sizes like 512, 640, 768, 896 and 1024. The new generated HERIDAL database will be of about 3000 images for training and validation, around 500 images for testing the model. The bigger the image size the more memory of GPU to train on deep CNNs. By proposing this new generated database we will overcome the problem of how to train huge aerial images in object or human detection and to find the bounding box accurately for testing the database. We have shown the difference of training with both 512 and 1024 aerial image sizes in the experiment section. Once we have the new database generated from the original HERIDAL database we will train our newly generated database on EfficientDET architecture [28] [29].

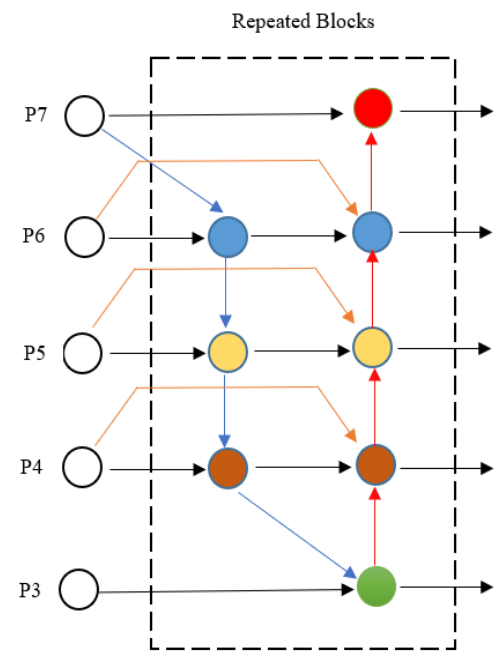

Figure 3: Bidirectional Feature Pyramid Network (BiFPN) 


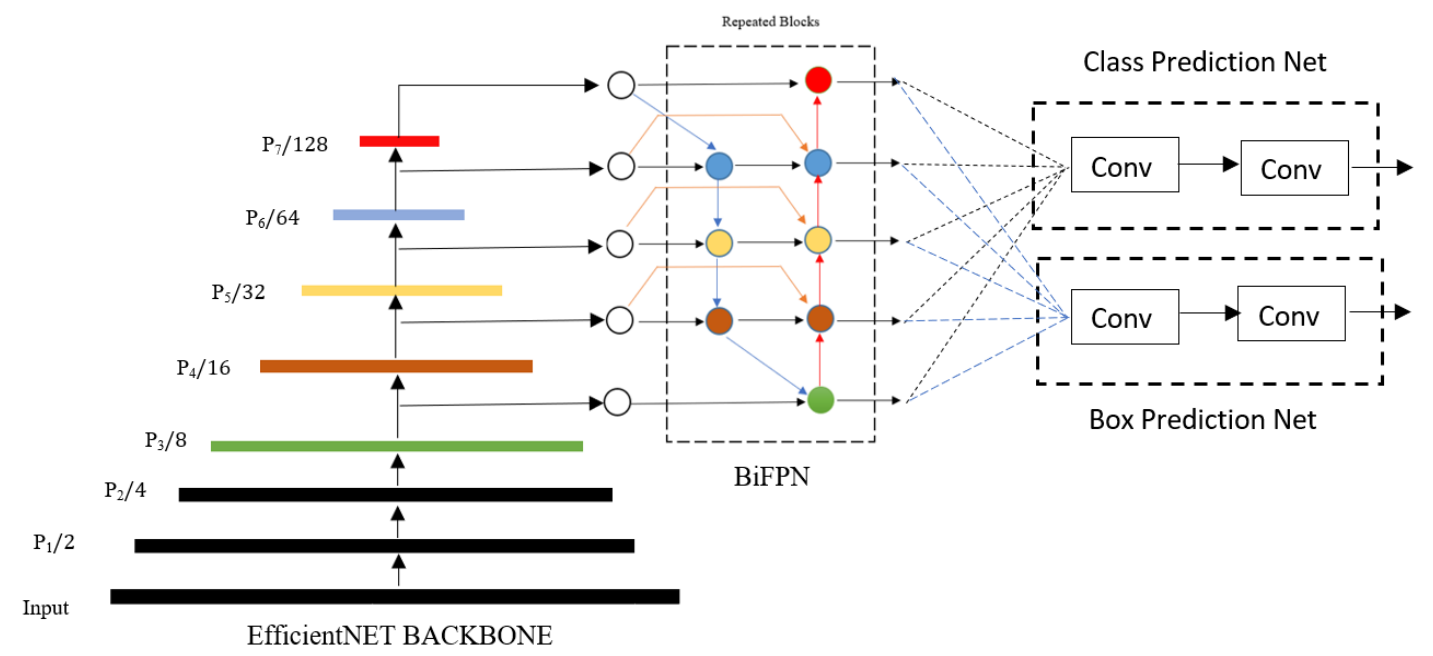

Figure 4: EfficientDET Architecture

EfficientDET has proven to have better results compared to yolov3 [26], Faster RCNN [24] and other existing famous object detectors. EfficientDET explains about the drawback of multi-scale feature fusion problem which can be solved by the use of BiFPN as feature network. As mentioned in EfficientDET paper [28], the architecture considers the fast normalized fusion over softmax based fusion as the softmax will slowdown the GPU hardware, whereas by using fast normalized fusion method the GPUs speed has increased by $30 \%$. EfficientDet also explains about the cross-scale connections with the existing pyramid networks like FPN [25], PANet [36], NAS-FPN [37]. EfficientDET is divided into two networks as Imagenet pre-trained EfficientNETs and repetitive BiPFN networks as the second layer as shown in fig 4. BiFPN layer is used to get the features from level 3 to level 7 of the backbone as shown in the figure 3 . These features are fed into class and box prediction networks as in fig 4 to identify the object classes in the image and to locate them precisely using bounding box. The repetitive BiFPN layers depends on the input size of the image which can be denoted by a compound coefficient $(\phi)$. Using this coefficient we can uniformly scale the network depth, width and resolution. Our generated dataset varies from 512, 640, 768, 896 and 1024 numerically from 0 to 4 on $\phi$ value. For example if the input size is 1024 the BiFPN layers will be seven, adding three with the $\phi$ value. So it's ideally versatile architecture when working with huge image resolution images or with drone images, as our generated dataset varies from 512 to 1024 by changing the value of scaling coefficient $(\phi)$. All the experimental results and comparisons are explained in the next section.

\section{EXPERIMENTS AND RESULTS}

We evaluate the pre-processed HERIDAL dataset [32] on EfficientDET architecture proposed by Tan M[28] [29]. The regenerated new HERIDAL dataset consists of about 3000 training and validation images in 512 image resolution. Testing of HERIDAL dataset have considered on approximately 500 images. In this section, we will discuss all the possible experiments and results based on tuning the architecture in both scenarios as per the fig. 2. We have divided our experiments into two sections, at first we train the repetitive BiFPN network by freezing the EfficientNET backbone using the pre-trained weights of the imagenet on the factors of batch size, epoch, batch normalization, phi $(\phi)$, optimizers and learning rate. In the second experiment we will train our model based on the same hyper tuning factors, but this time we will train our model on the whole network by unfreezing the backbone network. At first, We have trained our model on most of the existing optimizers like Adam, SGD, RMSprop, Adamax, Adadelta, Adagrad and Nadam and realized RMSprop, Nadam and SGD are faster in training and also show promising results compared to the others. We have compared our results with the state-of-art object detectors on HERIDAL dataset and also EfficientDET architecture with COCO Dataset [31] and VOC Dataset[33].

In fig. 5, we have trained our new generated HERIDAL dataset of $512 \times 512$ images on three different optimizers as Nadam, RMSProp and Adam by freezing the EfficientDET backbone which means we have trained on the repetitive BiFPN from level 3 to level 7 . The experiment has conducted by using batch normalization [38] and using the parameters of batch size 32, epoch 50, a step of 1000 and a learning rate of 0.001 . We can observe by using RMSprop, the results were quite promising and better compared to the other optimizers. we have achieved the accuracy of $91.27 \%$ mAP (Mean Average Precision) using RMSprop optimizer. The figure also shows the training loss of 0.55 and Validation loss of 1.4 for RMSProp optimizer. All the experiments have conducted by assuming the first scenario from the proposed method section i.e., two or more existing humans from the considered image size are generated as one single image.

For the next experiment, we unfreeze the EfficientDET backbone layer and train the whole network without batch normalization. We have set the hyper parameters of batch size 4, epoch 50, a step of 1000 and a low learning rate compared to the above experiment as 0.0001 . Here the results have been improved and proved to have better results compared to state-of-art paper [32] which had 

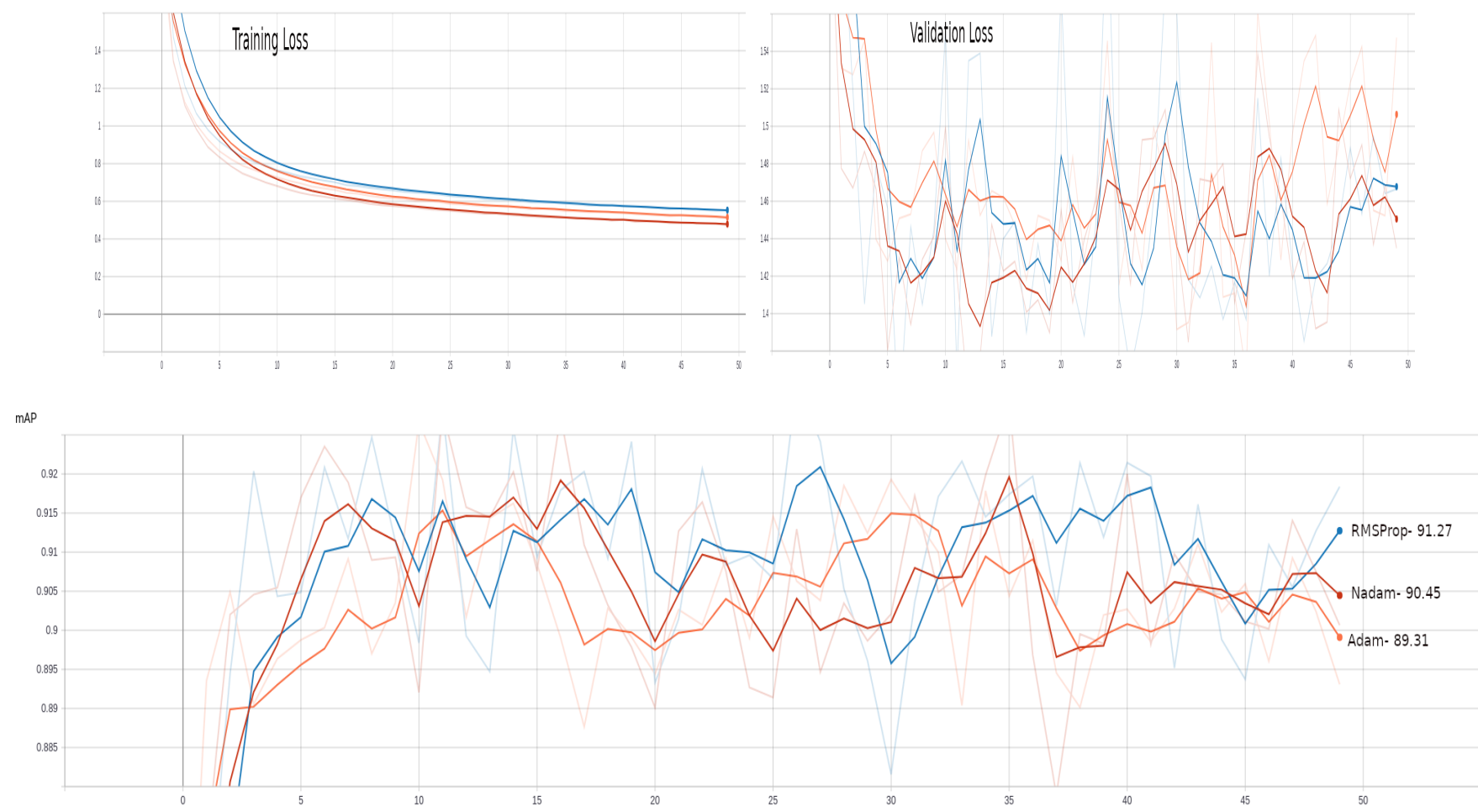

Figure 5: Experimental results using RMSprop (Blue), Nadam (Red) and Adam (Orange) on BiFPN
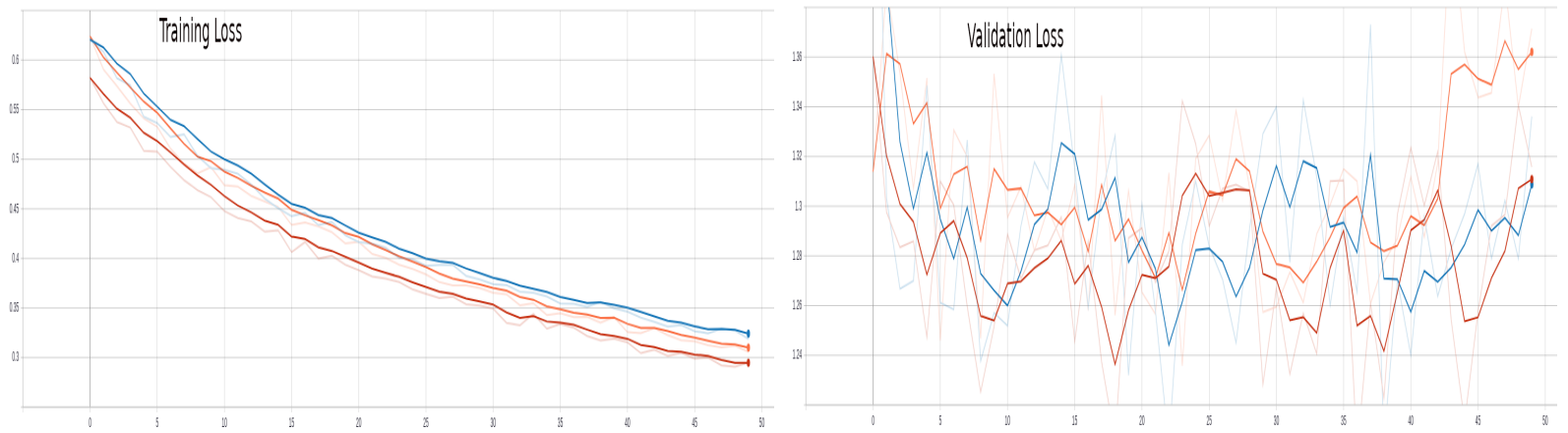

mAP

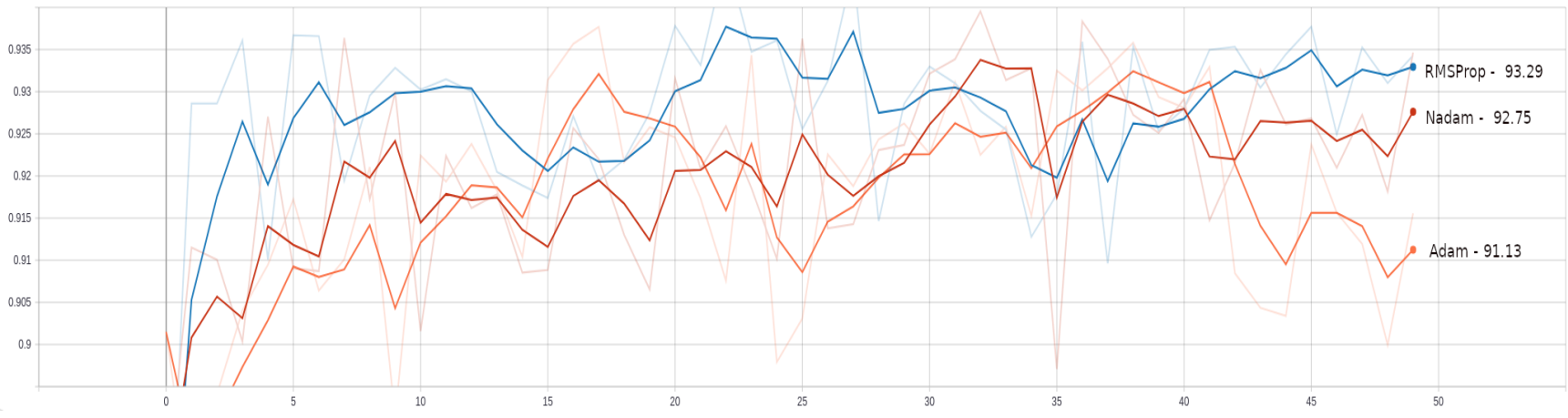

Figure 6: Experimental results using RMSprop (Blue), Nadam (Red) and Adam (Orange) on EfficientDET 


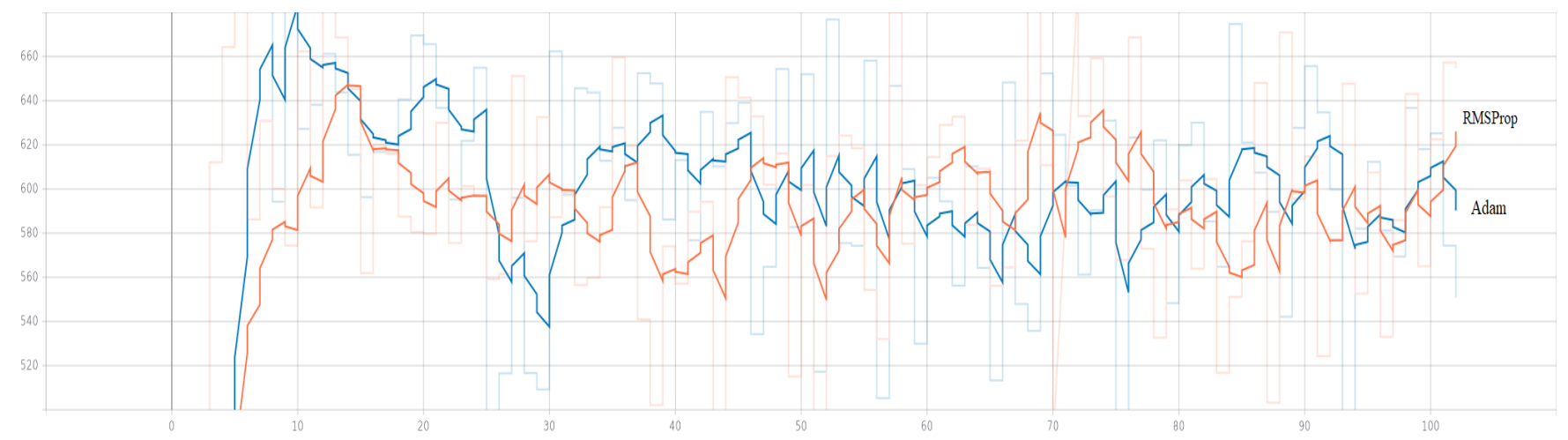

Figure 7: Experimental results using RMSprop (Orange) and Adam (Blue) on Yolov3
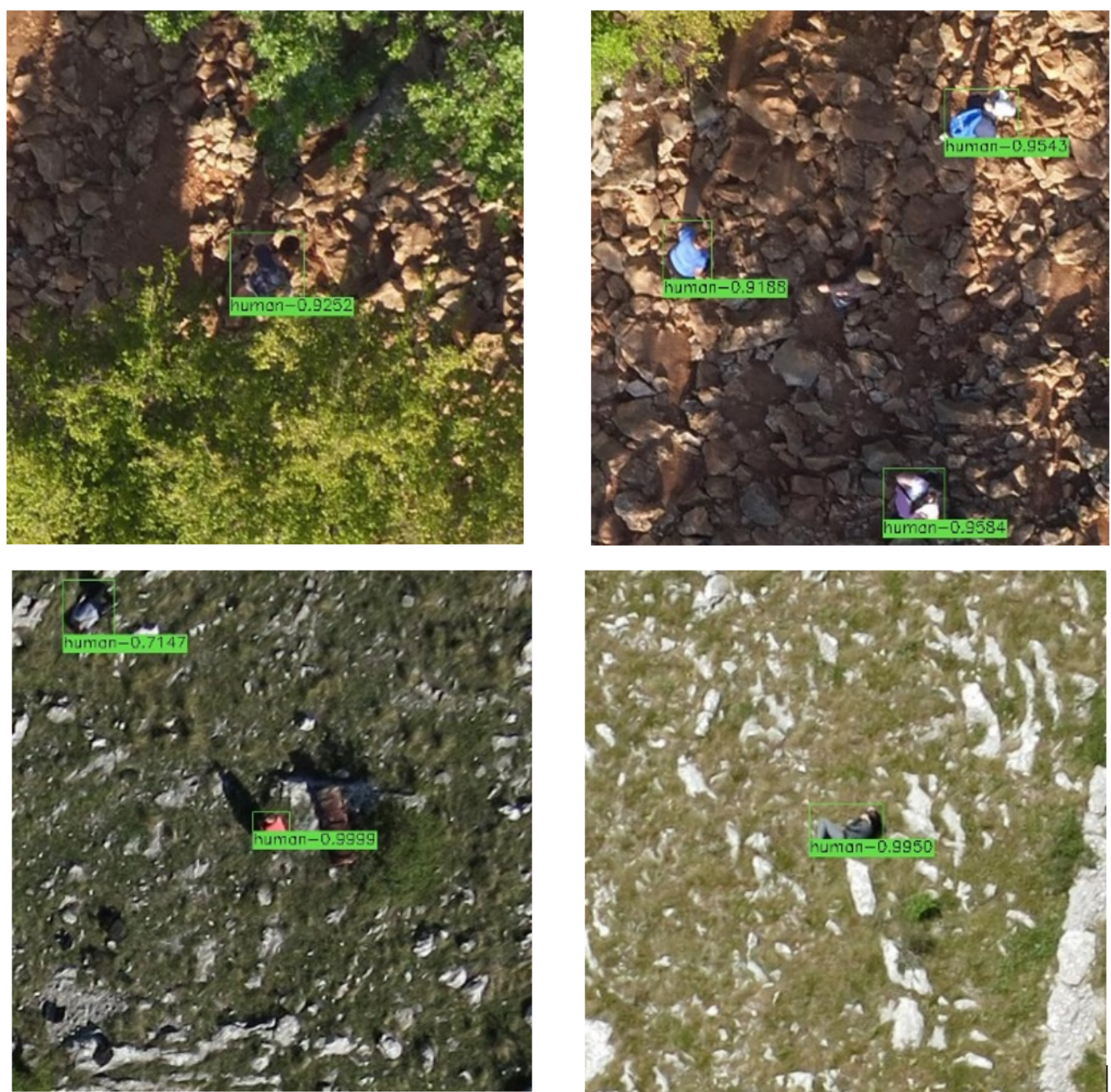

Figure 8: Detection of humans on the HERIDAL dataset[32] in different scenarios

achieved the accuracy of $88.9 \%$, while our results has achieved with an accuracy of $93.29 \%$ mAP using RMSprop optimizer. The results have been plotted in fig. 6 . We have also conducted experiments by changing the scaling coefficient $(\phi)$ from 0 to 4 which generates the images from 512 to 1024 resolution for the same optimizers with the same tuning parameters. But to train the dataset with image 
resolution of more than $512 \times 512$ a huge amount of GPU memory is required, so we have preferred on generated 512 image database. The proposed method has achieved the detection accuracy of $93.29 \%$ which proves to have better accuracy compared to the state-of-art proposed HERIDAL database paper which is based on extracting the salient features, which has slightly worse result of $88.9 \% \mathrm{mAP}$ compared to the proposed model in this paper.

In the next experiment, we have tested the proposed HERIDAL dataset on Yolov3 [26] with the existing optimizers adam and RMSprop by using the parameters as batch size 4, epoch 100 and a learning rate of 0.0001 . For Yolov3 [26] we have also chosen the parameter of maximum and minimum input size of the image. The results are plotted in the fig. 7 with an accuracy of $65.4 \%$ using RMSprop optimizer. In fig. 8, we can see a few of the detected humans from the new generated HERIDAL database. By solving the problem of detecting humans in Mediterranean images we can save many human lives who are struck over the mountains by search and rescue operations. The plotted results in this paper have the best accuracy compared to all other methods mentioned in the literature.

\section{CONCLUSION}

In this paper we have conducted experiments on the existing EfficientDET architecture with the new generated HERIDAL dataset explaining how the high resolution aerial images can be trained on the neural networks. And we have compared our results with the existing state-of-art papers on search and rescue operations and conclude we have the best accuracy.

\section{ACKNOWLEDGMENTS}

The work in this paper was funded by the European Union's Horizon 2020 research and innovation programme under the Marie Sklodowska-Curie grant agreement No 764951. We also gratefully acknowledge the support of NVIDIA Corporation with the donation of the Titan Xp GPUs used for this research.

\section{REFERENCES}

[1] J. Berclaz, F. Fleuret, E. Turetken and P. Fua, "Multiple Object Tracking Using KShortest Paths Optimization," IEEE Transactions on Pattern Analysis and Machine Intelligence, vol. 33, no. 9, pp. 1806-1819, Sept. 2011.doi: 10.1109/TPAMI.2011.21.

[2] K. Kang, H. Li, J. Yan, X. Zeng, B. Yang, T. Xiao, C. Zhang, Z. Wang, R. Wang, $\mathrm{X}$. Wang et al., "T-CNN: Tubelets with convolutional neural networks for object detection from videos," IEEE Transactions on Circuits and Systems for Video Technology, vol. 28, no. 10, pp. 2896-2907, 2018.

[3] P. Viola and M. Jones, "Rapid object detection using a boosted cascade of simple features," Proceedings of the 2001 IEEE Computer Society Conference on Computer Vision and Pattern Recognition. CVPR 2001, Kauai, HI, USA, 2001, pp. I-I. DOI: 10.1109/CVPR.2001.990517.

[4] Jones, Michael, and Paul Viola. "Fast multi-view face detection." Mitsubishi Electric Research Lab TR-20003-96 3.14 (2003): p.2.

[5] Turk, Matthew A., and Alex P. Pentland. "Face recognition using eigenfaces." Proceedings. 1991 IEEE Computer Society Conference on Computer Vision and Pattern Recognition. 1991, pp. 586-587.

[6] B. Hariharan, P. Arbelaez, R. Girshick, and J. Malik, "Simultaneous detection and segmentation," in European Conference on Computer Vision. Springer, 2014, pp. 297-312.

[7] Hariharan, Bharath, et al. "Hypercolumns for object segmentation and finegrained localization." Proceedings of the IEEE conference on computer vision and pattern recognition. 2015, pp. 447-456.

[8] K. He, G. Gkioxari, P. Dollar, and R. Girshick, "Mask R-CNN," in Computer Vision (ICCV), 2017 IEEE International Conference on. IEEE, 2017, pp. 2980-2988.

[9] Sun, Zehang, George Bebis, and Ronald Miller. "On-road vehicle detection: A review." IEEE transactions on pattern analysis and machine intelligence 28.5
(2006): pp. 694-711.

[10] Gotovac, Sven, Vladan Papić, and Željko Marušić. "Analysis of saliency object detection algorithms for search and rescue operations." 2016 24th IEEE International Conference on Software, Telecommunications and Computer Networks (SoftCOM), 2016, pp. 1-6.

[11] E. C. Fear, X. Li, S. C. Hagness and M. A. Stuchly, "Confocal microwave imaging for breast cancer detection: localization of tumors in three dimensions," in IEEE Transactions on Biomedical Engineering, vol. 49, no. 8, pp. 812-822, Aug. 2002. DOI: 10.1109/TBME.2002.800759.

[12] Serna J (2016) Lufthansa jet and drone nearly collide near lax. LA Times

[13] Jansen B (2015) Drone crash at white house reveals security risks. USA Today

[14] Shannon L (2019) DJI drones helped track and stop the Notre Dame fire. The Verge

[15] Zhao, Zhong-Qiu, et al. "Object detection with deep learning: A review." IEEE transactions on neural networks and learning systems 30.11 (2019): pp. 3212-3232.

[16] Rivera, A. J. A., et al. "Post-disaster rescue facility: Human detection and geolocation using aerial drones." 2016 IEEE Region 10 Conference (TENCON). IEEE, 2016, pp. 384-386.

[17] Yong, Suet-Peng, and Yoon-Chow Yeong. "Human Object Detection in Forest with Deep Learning based on Drone's Vision." 2018 4th International Conference on Computer and Information Sciences (ICCOINS). IEEE, 2018, pp. 1-5.

[18] Rudol, Piotr, and Patrick Doherty. "Human body detection and geolocalization for UAV search and rescue missions using color and thermal imagery." 2008 IEEE aerospace conference, 2008, pp. 1-8.

[19] Krizhevsky, Alex, Ilya Sutskever, and Geoffrey E. Hinton. "Imagenet classification with deep convolutional neural networks." Advances in neural information processing systems. 2012, pp. 1097-1105.

[20] Lowe, David G. "Distinctive image features from scale-invariant keypoints", International Journal of computer vision 60.2 (2004), pp. 91-110.

[21] McConnell, Robert K. "Method of and apparatus for pattern recognition." U.S. Patent No. 4,567,610. 28 Jan. 1986.

[22] P. Viola and M. J. Jones, "Robust real-time face detection", International Journal of computer vision, vol. 57, no. 2, pp.137-154, 2004

[23] Jiao, Licheng, et al. "A Survey of Deep Learning-Based Object Detection." IEEE Access 7 (2019), pp. 128837-128868.

[24] Ren, Shaoqing, et al. "Faster r-CNN: Towards real-time object detection with region proposal networks." Advances in neural information processing systems. 2015, pp. 91-99.

[25] Lin, Tsung-Yi, et al. "Feature pyramid networks for object detection." Proceedings of the IEEE conference on computer vision and pattern recognition. 2017, pp. $2117-2125$

[26] Redmon, Joseph, and Ali Farhadi. "Yolov3: An incremental improvement." arXiv preprint arXiv:1804.02767 (2018)

[27] Liu, Wei, et al. "SSD: Single shot multibox detector." European conference on computer vision. Springer, Cham, 2016, pp. 21-37.

[28] Tan, Mingxing, Ruoming Pang, and Quoc V. Le. "Efficientdet: Scalable and efficient object detection." Proceedings of the IEEE/CVF Conference on Computer Vision and Pattern Recognition. 2020, pp. 10781-10790.

[29] Tan, Mingxing, and Quoc V. Le. "Efficientnet: Rethinking model scaling for convolutional neural networks." arXiv preprint arXiv:1905.11946 (2019)

[30] Deng, Jia, et al. "Imagenet: A large-scale hierarchical image database." 2009 IEEE conference on computer vision and pattern recognition, 2009, pp. 248-255.

[31] T.-Y. Lin, M. Maire, S. Belongie, J. Hays, P. Perona, D. Ramanan, P. Dollar, and C. L. Zitnick, "Microsoft coco: Common objects in context," in European conference on computer vision. Springer, 2014, pp. 740-755.

[32] Božić-Štulić, Dunja, Željko Marušić, and Sven Gotovac. "Deep learning approach in aerial imagery for supporting land search and rescue missions." International Journal of Computer Vision 127.9 (2019), pp. 1256-1278.

[33] Everingham, Mark, et al. "The 2005 pascal visual object classes challenge." Machine Learning Challenges Workshop. Springer, Berlin, Heidelberg, 2005, pp. 117-176.

[34] Xiao, Han, Kashif Rasul, and Roland Vollgraf. "Fashion-mnist: a novel image dataset for benchmarking machine learning algorithms." arXiv preprint arXiv:1708.07747 (2017)

[35] Zhu, Pengfei, et al. "VisDrone-VDT2018: The vision meets drone video detection and tracking challenge results." Proceedings of the European Conference on Computer Vision (ECCV). 2018

[36] Liu, Shu, et al. "Path aggregation network for instance segmentation." Proceedings of the IEEE Conference on Computer Vision and Pattern Recognition. 2018, pp. 8759-8768

[37] Ghiasi, Golnaz, Tsung-Yi Lin, and Quoc V. Le. "Nas-fpn: Learning scalable feature pyramid architecture for object detection." Proceedings of the IEEE Conference on Computer Vision and Pattern Recognition. 2019, pp. 7036-7045.

[38] Ioffe, Sergey, and Christian Szegedy. "Batch normalization: Accelerating deep network training by reducing internal covariate shift." arXiv preprint arXiv:1502.03167 (2015) 